INRA Prod. Anim., 2008, 21 (3), 231-238

\title{
Le lapereau en développement : données comportementales, alimentaires et sensorielles sur la période naissance-sevrage
}

\author{
G. COUREAUD ${ }^{1}$, L. FORTUN-LAMOTHE2, H.-G. RÖDEL ${ }^{3}$, R. MONCLÚS $S^{3,4}$, B. SCHAAL \\ 1 CNRS, Université de Bourgogne, INRA, UMR 5170 Equipe Ethologie et Psychobiologie Sensorielle, \\ Centre Européen des Sciences du Goût, F-21000 Dijon, France \\ ${ }^{2}$ INRA, Université de Toulouse INP-ENSAT, ENVT, UMR 1289 Tissus Animaux, Nutrition, Digestion, \\ Ecosystème et Métabolisme, F-31326 Castanet-Tolosan, France \\ 3 Université de Bayreuth, Département de Physiologie Animale, Bayreuth, Allemagne \\ 4 Université Autonome de Madrid, Département de Biologie, Madrid, Espagne
} Courriel : coureaud@cesg.cnrs.fr

Tous les mammifères nouveau-nés doivent interagir rapidement avec leur mère pour ingérer du lait. Chez le lapin européen (Oryctolagus cuniculus) cela intervient dans un contexte d'interaction mère-jeunes limité dans le temps. La femelle met bas dans un nid isolé, dans lequel elle ne retourne qu'une brève ( $<5 \mathrm{~min}$ ) et unique fois par jour pour allaiter sa portée, après quoi elle le referme jusqu'au lendemain (Mykytowycz 1968, Broekhuizen et al 1986). Cela est aussi vrai chez les lapines de races domestiques, à l'exception de la fermeture du nid qui dépend de l'éleveur/animalier (Zarrow et al 1965, Hudson et Distel 1982, cf. Schulte et Hoy 1997 pour exception). Par ailleurs, même si elles n'ont pas les moyens de l'exprimer en conditions d'élevage, les femelles conservent dans leur répertoire comportemental le comportement de fermeture du nid en fin d'allaitement (elles l'expriment spontanément si elles sont replacées en conditions semi-naturelles, Deutsch 1957). Nous proposons ici une synthèse du développement du comportement du lapereau de la naissance au sevrage, en nous attachant plus particulièrement aux contraintes sociales et alimentaires auxquelles le jeune a à faire face.

\section{1 / Le comportement ali- mentaire du lapereau : du lait à l'alimentation solide}

Les lapines mettent bas après $31 \mathrm{j}$ de gestation. Les lapereaux entrent alors dans une période de développement rapide, aboutissant au sevrage en un mois environ. Les jeunes connaissent donc un basculement progressif de leur alimentation, d'exclusivement lactée et délivrée par la mère, à solide et choisie de façon autonome.

\section{1 / Prise de lait}

La première tétée intervient dès la naissance. Arc-boutée au dessus de sa portée, la femelle demeure relativement immobile, ne fournissant pas d'aide directe aux lapereaux (Hudson et Distel 1982). La localisation des tétines et la prise de lait dépendent donc fortement du comportement des lapereaux. Dans ce contexte, les nouveau-nés sont généralement très efficaces, puisqu'ils parviennent à ingérer jusqu'à 25\% de leur poids en lait ( 5 à $10 \mathrm{~g}$ le jour de la naissance). Il n'est cependant pas rare qu'un à deux lapereaux par portée ne prennent pas de lait lors d'un allaitement (soit 10-15\% des lapereaux à J1, Coureaud et al 2007). Les tétées des 2-3 premiers jours sont en tout cas absolument critiques pour la survie : en manquer deux successivement aboutit souvent à la mort (Coureaud et al 2000). Les lapereaux n'expriment pas de choix sélectif des tétines, mais en changent fréquemment au cours d'un même allaitement. Pourtant, la compétition est forte sous la mère (du fait, pour les races domestiques, d'un nombre de lapereaux généralement plus élevé que celui de tétines disponibles, Drummond et al 2000, Bautista et al 2005). L'absence de préférence pour une tétine offre donc une chance à chaque nouveau-né de téter lorsque la femelle est présente. La prise individuelle de lait augmente de 5-10 g/j à la naissance jusqu'à environ $30 \mathrm{~g} / \mathrm{j}$ à $\mathrm{J} 20$ 25 . Au cours de cette période, elle varie fortement entre lapereaux et portées, en fonction des capacités sensori-motrices des lapereaux, de leurs aptitudes à lutter dans un environnement compétitif, et de la production laitière de la femelle (Fortun-Lamothe et Gidenne 2000, Rödel et al 2008a). Au-delà de J25 et jusqu'au sevrage, la production de lait décroît. La femelle est de nouveau fertile dès les jours qui suivent la mise bas, et peut supporter simultanément la lactation et une nouvelle gestation. Dans ce cas, la production de lait cesse 2-3 j avant la mise bas suivante (Lebas 1972, Fortun-Lamothe et al 1999). En conditions d'élevage, le sevrage est généralement réalisé entre J28 et J35. En conditions naturelles, la lactation peut durer de 4 à 6 semaines, si les ressources alimentaires sont suffisantes pour la mère et que celle-ci n'est pas gestante ; si la femelle est gestante, les lapereaux peuvent être sevrés dès 3 semaines (Broekhuizen et al 1986).

\section{2 / Prise d'aliment solide}

Les lapereaux commencent à s'alimenter solidement dès J16-18, lorsqu'ils sont aptes à sortir du nid et à accéder à la mangeoire maternelle. Cependant, les premiers contacts avec des éléments non lactés interviennent dans les jours qui suivent la naissance (grignotage des pelotes fécales déposées par la mère dans le nid). Au début, les lapereaux n'ingèrent que peu de granulés $(<2 \mathrm{~g} / \mathrm{j}$ avant $\mathrm{J} 20)$, mais cela augmente fortement dès $\mathrm{J} 25$ pour atteindre 40-50 g/j au sevrage (Gidenne et Fortun-Lamothe 2002). En élevage, 
la quantité d'aliment solide et d'eau ingérée excède celle de lait au cours de la $4^{\text {ème }}$ semaine postnatale (Scapinello et al 1999, Gidenne et Lebas 2006). La caecotrophie semble démarrer aux alentours de J21-25 (Gidenne et al 2002).

\section{3 / Besoins nutritionnels}

Parallèlement aux modifications qui s'opèrent dans le comportement alimentaire du lapereau en développement, le besoin en nutriments évolue (figure 1). Le lait est riche en lipides $(13 \%)$ et protéines $(12 \%)$, et faible en lactose (Maertens et al 2006). A l'inverse, les granulés solides contiennent beaucoup de glucides (80\%), un niveau équivalent de protéines (15$18 \%$ ) et peu de lipides (2-5\%). Des modifications des capacités digestives du jeune vont de pair avec ces changements (Gidenne et Fortun-Lamothe 2002). Autour de J25, la quantité de protéines apportée par les granulés atteint celle apportée par le lait, puis la dépasse dans les jours qui suivent. Les lipides, eux, proviennent essentiellement du lait jusqu'au sevrage. Alors que l'apport de glucides est quasi nul jusqu'à $\mathrm{J} 17 \quad(<0,3 \mathrm{~g} / \mathrm{j})$, il devient notable à partir de $\mathrm{J} 21$ sous la forme de fibres et d'amidon. Cependant, les protéines du lait et les graisses constituent la source principale d'énergie jusqu'au sevrage.

Figure 1. Evolution de la composition en nutriments de l'alimentation consommée par les lapereaux élevés en conditions d'élevage entre la naissance et le sevrage.

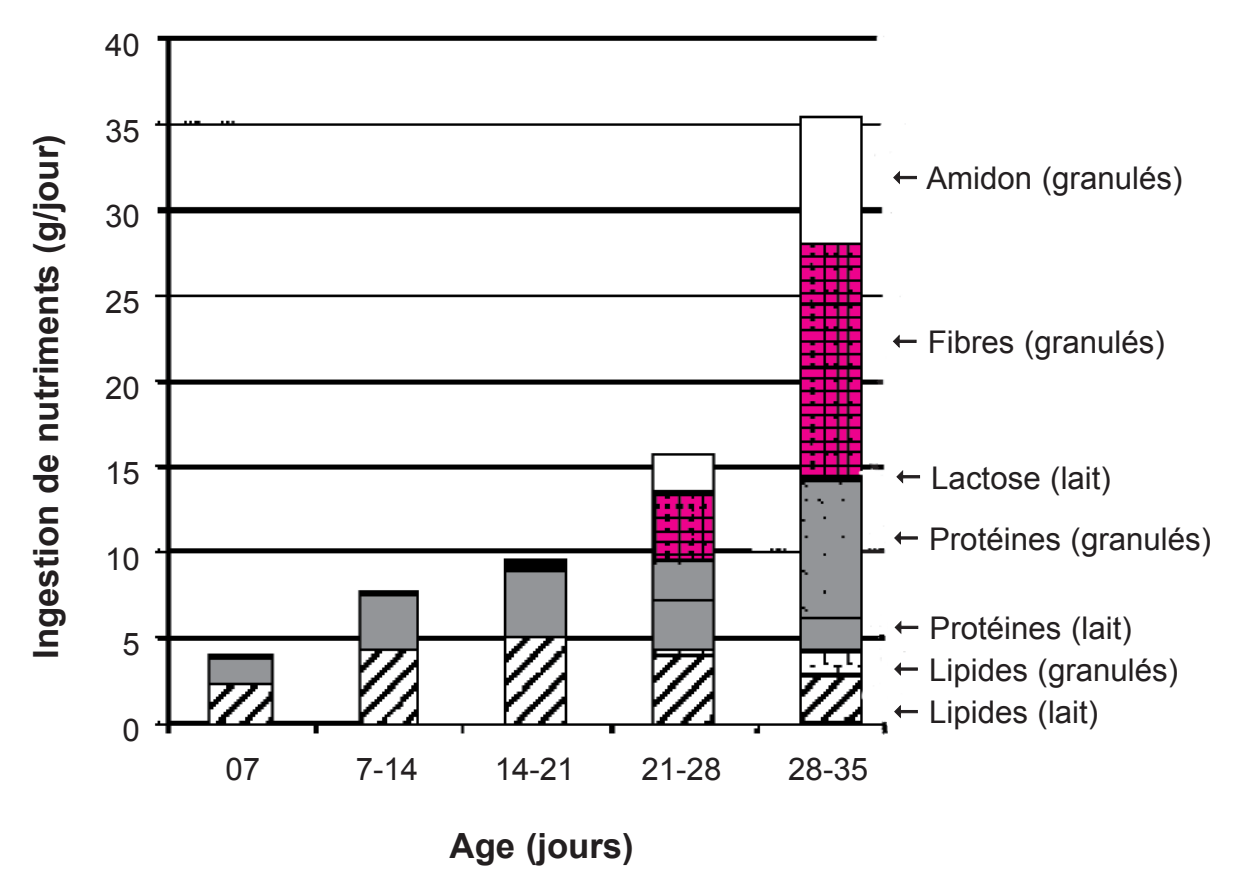

Age (jours)

\section{4 / Modulation du début de l'alimentation solide}

Si la taille de la portée (6-12 lapereaux en élevage) est fortement réduite et/ou blement, le début de l'alimentation solide peut être repoussé de $2-4 \mathrm{j}$ (FortunLamothe et Gidenne 2000). Offrir une deuxième possibilité quotidienne de téter (auprès d'une autre femelle) peut aussi décaler de plusieurs jours la première prise d'aliment solide (Gyarmati et al 2000). A l'inverse, le sevrage précoce $(<\mathrm{J} 25)$ accélère grandement le début de l'ingestion de granulés (Gallois et al 2005, Xiccato et al 2005). Le mode de présentation de la nourriture, et la taille et qualité des granulés peuvent aussi jouer un rôle (Maertens et Villamide 1998).

\section{2 / Facteurs influençant le développement du lapereau en conditions naturelles et semi-naturelles}

Cantonnés au nid jusqu'à ce que les yeux s'ouvrent (peu après J10), les lapereaux commencent à s'approcher de la mère à l'entrée du terrier à compter de J15-16, lorsqu'elle l'ouvre, mais ce n'est que vers J20-22 qu'ils en émergent vraiment, lorsque la femelle le laisse ouvert (Mykytowycz et Rowley 1958, Broekhuizen et al 1986, Rödel observations personnelles). que la production de lait augmente sensi-

\section{4 / Histoire reproductive de la mère}

Du fait de l'oestrus post-partum, les femelles donnent généralement naissance à des portées successives, dès le printemps (Rödel et al 2008b). Les lapereaux des premières portées de l'année semblent alors avoir un taux de croissance plus élevé que ceux des portées suivantes, ce qui suggère un impact défavorable du fait d'être à la fois allaitante et gestante. Mais globalement, cet effet serait plus faible que celui de la taille de portée et de l'âge de la mère (Rödel et al 2008a).

\section{5 / Température}

Les lapereaux vivant en conditions naturelles, doivent faire face à des 
Figure 2. Gain de poids moyen ( $\pm S D$ ) par tétée de lapereaux ( $n=172$, de 48 portées) d'âges croissants vivant en conditions semi-naturelles. L'échantillon d'animaux est donné entre parenthèses pour chaque âge. Les groupes sont indépendants d'un âge à l'autre (Rödel données personnelles).

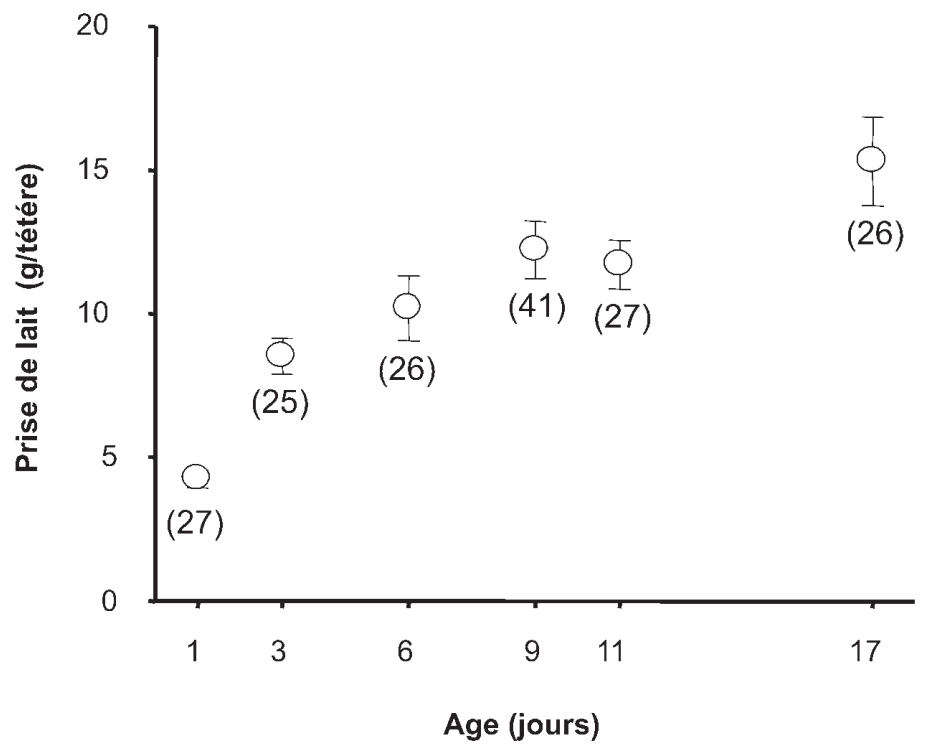

écarts thermiques quotidiens importants. Ces écarts peuvent altérer leur métabolisme et jouer sur leur croissance. Par exemple, le taux de croissance de portées de taille moyenne apparaît plus élevé en environnement froid que celui de portées de petite taille, malgré la plus forte compétition pour la tétée (Rödel et al 2008b). Ceci résulterait du bénéfice thermique tiré de la présence d'un plus grand nombre d'individus dans la fratrie.

\section{3 / Développement sensori- moteur du lapereau}

Les capacités sensori-motrices du lapereau à la naissance, bien que limitées, sont hautement spécialisées. Elles vont rapidement évoluer jusqu'au sevrage, parallèlement aux besoins sociaux et alimentaires du jeune.

Semaine 1 : Dépendance totale de l'olfaction et de la somesthésie. Les lapereaux naissent nus, dépourvus d'audition et de vision, et leurs aptitudes motrices sont limitées. Cependant, leurs capacités olfactives et somesthésiques fonctionnent depuis la vie foetale, ce qui leur permet de répondre à la naissance aux stimulations thermotactiles (Pacheco-Cobos et al 2003) et aux gradients d'odeurs émanant du corps maternel (Schley 1979, Hudson et Distel 1983, Coureaud et Schaal 2000, Coureaud et al 2001a). Les capacités sensori-motrices des nouveau-nés leur permettent par ailleurs, en complément de l'existence d'une horloge interne se

mettant en place au cours des premiers jours postnatals, d'exprimer un rythme d'activité quotidien, calé sur la tétée. Cela les amène à émerger des profondeurs du nid, à être en état d'éveil maximal, et ainsi à anticiper l'arrivée de la femelle, juste avant qu'elle ne vienne allaiter (Hudson et Distel 1982, Jilge 1993, Bautista et al 2003). Durant cette semaine, le système masticateur n'est pas encore mature, mais les lapereaux peuvent grignoter des éléments du nid et les pelotes fécales déposées dans ce dernier par la femelle (Mykytowycz et Ward 1971, Kovacs et al 2004, Moncomble et al 2004).

Semaine 2 : Emergence de l'audition, de la vision et de la mastication. Les lapereaux commencent à entendre à $\mathrm{J} 7-8$, puis à voir vers $\mathrm{J} 10-13$, en même temps que leurs capacités masticatrices apparaissent (Gottlieb 1971). Au terme de la deuxième semaine, ils commencent à sortir du nid et à consommer, en élevage, les premiers granulés. Le rôle de l'olfaction par rapport à la vision dans ces premières sorties hors nid reste à évaluer. En élevage, les lapereaux se mettent progressivement à solliciter la mère pour téter, ce qui peut aboutir au harassement de la lapine qui finit par chercher à s'isoler des jeunes (hors allaitement, Mirabito et al 2004).

Semaine 3 : Importance grandissante de la vision. Au terme de la troisième semaine, la production de lait par la mère atteint son maximum, de même que la consommation de lait par le lapereau. En élevage, les lapereaux quittent définitivement le nid, localisent la mère par la vue, et initient la tétée presque systématiquement. Ils augmentent progressivement leur prise d'aliment solide et d'eau, même si celle de lait demeure supérieure jusqu'à J25 (Langenbach et al 1992, Scapinello et al 1999). En conditions naturelles, les lapereaux viennent de plus en plus à l'entrée du terrier, où ils sont allaités (Broekhuizen et Mulder 1983, Rödel données non publiées).

Semaine 4 : Sevrage. L'équipement sensoriel est maintenant suffisamment mature pour permettre de faire face aux besoins de la vie autonome. Comme les femelles cessent progressivement d'allaiter, les lapereaux sont obligés de s'orienter vers des aliments solides. Cela peut s'accompagner de comportements agressifs exprimés par la mère envers ses jeunes, les conduisant à s'éloigner (Mykytowycz et Ward 1971).

\section{4 / Communication olfacti- ve entre la mère et les jeu- nes}

Nous résumons ici certains résultats obtenus au cours des dernières décennies, qui ont contribué à mettre en avant l'effet de certaines odeurs maternelles sur le comportement du lapereau,

\section{1 / Odeurs apprises avant la naissance}

Comme chez de nombreuses espèces (Schaal et Orgeur 1992, Schaal et al 2001), les fotus lapins détectent et apprennent des odeurs, qui sont alors attractives après la naissance. Ainsi, des lapereaux nés de femelles ayant mangé du cumin pendant la gestation s'orientent préférentiellement vers cette odeur et vers des placentas qui la contiennent (Coureaud et al 2002). Ce phénomène s'observe avec d'autres arômes (Semke et al 1995, Coureaud et al 2002). Par ailleurs, les lapereaux nés de femelles au régime enrichi en cumin, préfèrent également le lait de ces femelles à du lait de femelles au régime témoin (Coureaud $e t$ al 2002). Ils détectent donc dans le placenta comme dans le lait, des indices odorants communs qui forment une continuité chimique entre les environnements pré- et postnatal. Cette continuité chimique contribue à l'orientation rapide et efficace du nouveau-né vers la mère et le lait. D'ailleurs, si elle est expérimentadont le comportement de tétée. 
lement interrompue, les lapereaux tètent moins bien lors des premiers allaitements (Coureaud et al 2002).

\section{2 / L'odeur du lait de lapine}

Les nouveau-nés répondent à l'odeur du lait car ils y détectent pour partie des odeurs familières, notamment apprises avant la naissance et liées à l'alimentation de la mère (voir ci-dessus). Mais comme ils répondent également au lait de toute femelle de l'espèce, indépendamment de son régime alimentaire (Coureaud 2001, Coureaud et al 2002), on doit en déduire qu'ils y détectent aussi des odeurs communes à l'ensemble des femelles Oryctolagus allaitantes. Parmi ces dernières, un composé volatile monomoléculaire a été isolé et identifié dans le lait, le 2-méthyl2-buténal (2M2B, Coureaud 2001, Schaal et al 2003). Il déclenche le comportement de recherche-saisie orale, impliqué dans la localisation des tétines et la tétée, chez plus de $90 \%$ des nouveau-nés (dans une gamme précise de concentration, Coureaud et al 2004). Testé quant à ses propriétés phéromonales, sur la base d'une définition rigoureuse du concept comprenant 5 critères précis (Beauchamp et al 1976), ce signal 1) chimiquement simple, est à lui seul aussi actif sur le comportement du lapereau que l'odeur complexe (> 150 composés) du lait ; 2) déclenche des réponses comportementales similaires (attraction, recherche-saisie orale) ayant une fonction adaptative pour les lapereaux, dans le sens où elles contribuent à l'interaction avec la mère et au succès de tétée ; 3) présente une activité sélective (non déclenchée par n'importe quelle odeur) ; 4) a un impact spécifique à l'espèce, c'est-à-dire exclusif à $O$. cuniculus (seule espèce subsistante du genre Oryctolagus ; la présentation à des levreaux, par exemple, n'engendre aucune réponse) ; 5) a une activité apparemment indépendante de tout apprentissage préalable (y compris prénatal). C'est parce que le $2 \mathrm{MB} 2$ satisfait à ces 5 critères qu'il a été qualifié de phéromone. Paraissant principalement produit ou libéré dans la mamelle (Moncomble et al 2005), il a été nommé «phéromone mammaire» (Coureaud 2001, Coureaud et al 2003, Schaal et al 2003 ; d'autres signaux phéromonaux sont suspectés intervenir dans la relation mère-jeunes chez le lapin, mais leur nature chimique reste à identifier, Hudson et Distel 1983, Keil et al 1990).

\section{3 / Un signal à l'activité modu- lée entre la naissance et le sevra- ge}

L'impact de la Phéromone Mammaire (PM) est extrêmement fort chez les nouveau-nés, dès les minutes qui suivent la naissance, y compris chez les prématurés (Coureaud 2001, Schaal et al 2003). Le fait que la PM déclenche des comportements orientés vers la mère et ses tétines suggère que son rôle principal est de contribuer au succès de tétée. Cette hypothèse a été testée en conditions d'élevage : les lapereaux qui ne répondaient pas à la $\mathrm{PM}(<9 \%)$ ingéraient moins de lait que ceux qui y répondaient, en particulier chez les lapereaux de petits poids $(<48 \mathrm{~g})$. La mortalité des non-répondants était par ailleurs plus élevée que celle des répondants sur la période J1-21 (figure 3a, Coureaud et al 2007, Fortun-Lamothe et al 2007). La réponse précoce à la PM serait donc un critère de viabilité des lapereaux, au même titre que le poids de naissance et le gain de poids.

Cependant, l'activité de la PM évolue au cours du développement. Elle est régulée par au moins deux facteurs. Premièrement, alors qu'ils répondent de façon quasi automatique à la PM à la naissance, les lapereaux y répondent en fonction de leur état de faim dès J5, leur réponse étant plus forte avant comparé à après la tétée (Montigny et al 2006). Deuxièmement, alors que la réponse de recherche-saisie orale à la PM est maximale avant l'allaitement quotidien pendant la 1 ère semaine, celle-ci décroît lorsque les yeux s'ouvrent, plus encore lorsque les lapereaux quittent le nid et débutent leur alimentation solide, et s'annule au sevrage (figure 3b, Coureaud et al 2008a). Cette évolution est assez similaire chez les lapereaux élevés en conditions semi-naturelles, si ce n'est que leur réponse s'annule plus tôt (figure 3b). Quoi qu'il en soit, indépendamment de leur génotype et des conditions écologiques dans lesquelles ils se développent (nature ou élevage), les lapereaux répondent toujours très fortement au signal au cours des premiers jours qui suivent la naissance. Ceci renforce l'idée selon laquelle la PM serait un signal biologique maternel essentiellement actif comme messager chimique, facilitateur de la prise lactée et de la survie des nouveau-nés. Il n'est cependant pas exclu que la PM conserve une signification au-delà du sevrage, comme l'ont montré des résultats préliminaires suggérant un effet sur l'appétence de l'aliment solide enrichi en PM, ainsi qu'une mortalité plus faible des animaux nourris avec cet aliment (Coureaud et al 2001b).

\section{4 / Un signal qui permet l'ap- prentissage de nouvelles odeurs}

Les lapereaux ont besoin d'apprendre rapidement certaines informations présentes dans leur environnement, tant pour optimiser leur adaptation à cet environnement que pour se préparer aux environnements futurs. Ils y parviennent dès la vie utérine (Bilkó et al 1994, Semke et al 1995, Coureaud et al 2002). Cela se poursuit à la naissance, les lapereaux pouvant apprendre des

Figure 3. a. Gain de poids à $J 1$ et mortalité sur la période J1-7 de lapereaux non répondants (Non $R$ ) ou répondants $(R)$ à la phéromone mammaire à $J 1$; b. Réactivité à la phéromone mammaire de lapereaux élevés en conditions d'élevage ou en conditions semi-naturelles entre J0 (naissance) et J28 (d'après Coureaud et al 2007, 2008a).

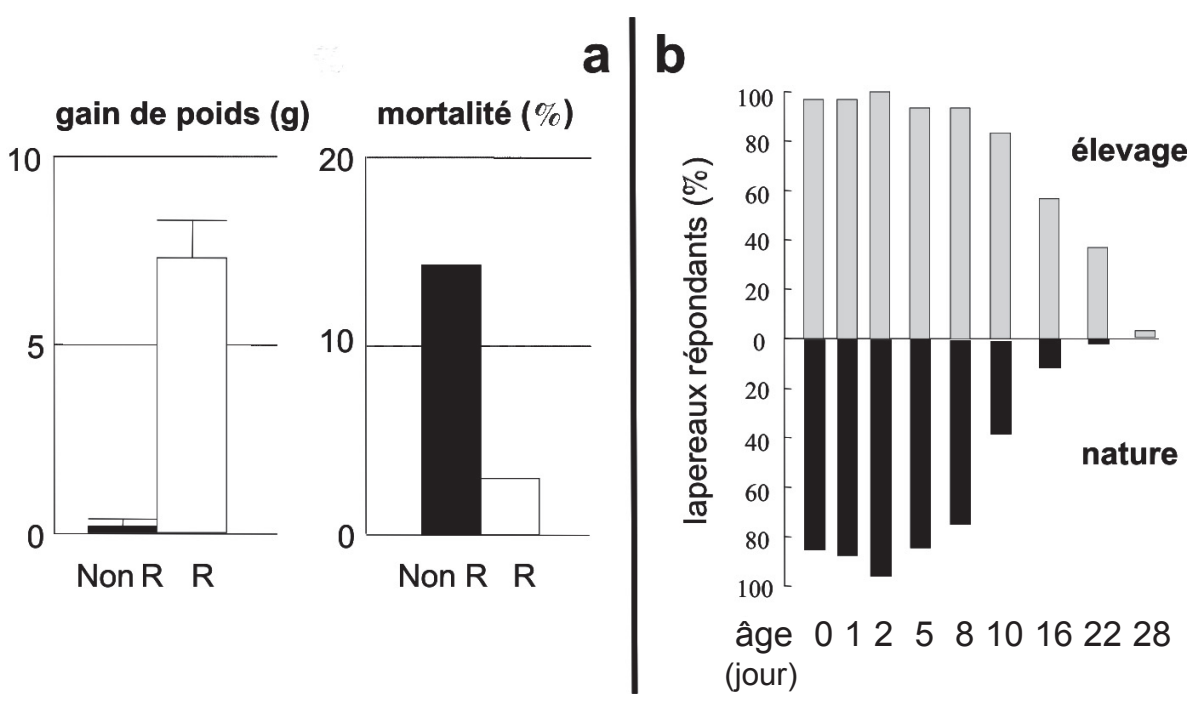


odeurs liées au nid (Hudson 1993 , Hudson et al 2003, Serra et Nowak 2005) ou présentes sur le corps de la mère (par exemple : Ivanitskii 1962, Kindermann et al 1991, Coureaud et al 2006). Récemment, il a été montré que la phéromone mammaire elle-même engendrait l'apprentissage d'odeurs : lorsque des nouveau-nés sont exposés à la PM en même tant qu'ils le sont à une odeur nouvelle, ils apprennent cette dernière grâce à la $\mathrm{PM}$ et y répondent $24 \mathrm{~h}$ plus tard par le comportement typique de tétée (Coureaud et al 2006). La PM peut ainsi faire apprendre très vite des odeurs aux nouveau-nés, dont des odeurs portées par la mère auxquelles ils sont exposés au cours de l'allaitement quotidien. Cela peut potentiellement optimiser la détection par les lapereaux de l'arrivée de la mère dans le nid et leur aptitude à localiser les tétines, et contribuer à la reconnaissance de la mère (Patris et al 2008) ainsi qu'au développement de préférences sociales et alimentaires utiles après le sevrage (Montigny et al 2007).

\section{Conclusion}

Pour assurer la pérennité de l'espèce, les mères et jeunes lapins ont développé des mécanismes sensoriels, physiologiques, cognitifs et comportementaux optimisant leurs échanges au cours de leur brève rencontre quotidienne. Ils présentent en particulier des mécanismes facilitant le transfert et l'ingestion de lait, indispensables à la survie et la croissance du jeune. L'olfaction y joue un rôle crucial. Elle contribue notablement à l'orientation efficace du nouveau-né vers le corps de la mère et à sa localisation rapide des tétines. Durant cette période de développement à haut risque pour le jeune, la mère communique avec ce dernier grâce à des odeurs individuelles (odeurs liées à son alimentation, par exemple) ou communes à l'ensemble des femelles allaitantes de l'espèce. La phéromone mammaire constitue un tel signal spécifique, hautement réactogène pour le nouveauné, dont l'activité remarquable consiste à la fois à déclencher des comporte- ments néonatals impliqués dans la tétée, et à faciliter l'apprentissage rapide d'informations nouvelles par le lapereau en quête de tels repères. Des expériences en cours cherchent à comprendre comment, et pour combien de temps les lapereaux détectent et répondent aux odeurs émises par la mère ou à celles apprises à son contact. Ces odeurs pourraient en effet jouer un rôle clé non seulement dans la survie du nouveau-né, mais aussi dans le développement et le bien-être du lapin postsevré.

\section{Remerciements}

Nous remercions sincèrement le Centre National de la Recherche Scientifique (CNRS), le Ministère de l'Enseignement Supérieur et de la Recherche, l'INRA, les Conseils Régionaux de Bourgogne et de PoitouCharente, ainsi que la Fondation Fyssen pour les appuis financiers accordés à nos travaux.

\section{Références}

Bautista A., Drummond H., Martina-Gomez M., Hudson R., 2003. Thermal benefit of sibling presence in the newborn rabbit. Dev. Psychobiol., 43, 208-215.

Bautista A., Mendoza-Degante M., Coureaud G., Martina-Gomez M., Hudson R., 2005. Scramble competition in newborn domestic rabbits for an unusually limited milk supply. Anim. Behav., 70, 997-1002.

Beauchamp G.K., Doty R.L., Moulton D.G., Mugford R.A., 1976. The pheromone concept in mammals: A critique. In: Mammalian olfaction, reproductive processes and behavior. Doty R.L. (Ed), Academic Press, New York, USA, 143160.

Bilkó A., Altbäcker V., Hudson R., 1994 Transmission of food preference in the rabbit: the means of information transfer. Physiol. Behav., 56, 907-912.

Broekhuizen S., Mulder J.L., 1983. Differences and similarities in nursing behaviour of hares and rabbits. Acta Zool. Fennica, 174, 61-63.

Broekhuizen S., Bouman E., Went W., 1986. Variations in timing of nursing in the brown hare (Lepus europaeus) and the european rabbit (Oryctolagus cuniculus). Mamm. Rev., 16, 139144.

Coureaud G., 2001. La régulation olfactive de la prise lactée chez le lapereau : caractérisation éthologique et chimique d'un signal phéromonal. Thèse de Doctorat, Université Paris 13, Paris, France, $215 p$.

Coureaud G., Schaal B., 2000. Attraction of newborn rabbits to abdominal odors of adult conspecifics differing in sex and physiological state. Dev. Psychobiol., 36, 271-281.

Coureaud G., Schaal B., Coudert P., Rideaud P., Fortun-Lamothe L., Hudson R., Orgeur P., 2000. Immediate postnatal suckling in the rabbit: its influence on pup survival and growth. Reprod. Nutr. Dev., 40, 19-32.

Coureaud G., Schaal B., Langlois D., Perrier G., 2001a. Responsiveness of newborn rabbits to surface odour cues from females differing in lactational state and to milk. Anim. Behav., 61, 153162.

Coureaud G., Langlois D., Fortun-Lamothe L., Schaal B., 2001b. Composition pour l'alimentation des lapins. Brevet 0102752 CNRS-INRA.

Coureaud G., Schaal B., Hudson R., Orgeur P. Coudert P., 2002. Transnatal olfactory continuity in the rabbit: behavioral evidence and short-term consequence of its disruption. Dev. Psychobiol., $40,372-390$

Coureaud G., Langlois D., Perrier G., Schaal B., 2003. Le 2M2B : une phéromone qui facilite la tétée du lapereau. Sem. Vét., 1117, 39-40.

Coureaud G., Langlois D., Sicard G., Schaal B., 2004. Newborn rabbit reactivity to the mammary pheromone: concentration-response relationship. Chem. Senses, 29, 341-350.

Coureaud G., Moncomble A.S., Montigny D. Dewas M., Perrier G., Schaal B., 2006. A pheromone that rapidly promotes learning in the newborn. Curr. Biol., 16, 1956-1961.

Coureaud G., Fortun-Lamothe L., Langlois D., Schaal B., 2007. The reactivity of neonatal rabbits to the mammary pheromone as a probe for viability. Animal, 1, 1026-1032.
Coureaud G., Rödel H.G., Kurz C.A., Schaal B., 2008a. Comparison of rabbit pup responsiveness to the mammary pheromone in breeding and semi-wild conditions. Chemoecology, 18, 53-59.

Coureaud G., Fortun-Lamothe L., Rödel H., Monclús R., Schaal B., 2008b. Development of social and feeding behaviour in young rabbits. Xiccato G., Trocino A., Lukefahr S.D. (Eds) Proc. 9th World Rabbit Cong., June 10-13, Verona, Italy, 1131-1146.

Deutsch J.A., 1957. Nest building behaviour of domestic rabbits under semi-natural conditions. Brit. J. Anim. Behav., 2, 53-54.

Drummond H., Vázquez E., Sánchez-Colón S., Martínez-Gómez M., Hudson R., 2000. Competition for milk in the domestic rabbit: survivors benefit from littermate deaths. Ethology, $106,511-526$

Fortun-Lamothe L., Gidenne T., 2000. Effects of the suckled litter size on intake behaviour, performance and health status of young and reproducing rabbits. Ann. Zootech., 49, 517-529.

Fortun-Lamothe L., Prunier A., Bolet G., Lebas F., 1999. Physiological mechanisms involved in the effects of concurrent pregnancy and lactation on fotal growth and survival in the rabbit. Livest. Prod. Sci., 60, 229-241.

Fortun-Lamothe L., Coureaud G., Langlois D., Schaal B., 2007. La réactivité des lapereaux à la phéromone mammaire : un indicateur de viabilité ? 12èmes Journ. Rech. Cunicole, 27-28 Novembre, Le Mans, France, 7-10.

Gallois M., Gidenne T., Fortun-Lamothe L., Le Huerou-Luron I., Lallès J.P., 2005. An early stimulation of solid feed intake stimulation 
slightly influences the morphological gut maturation in the rabbit. Reprod. Nutr. Dev., 45, 109-122.

Gidenne T., Fortun-Lamothe L., 2002. Feeding strategy for young rabbits around weaning: a review of digestive capacity and nutritional needs. Anim. Sci., 75, 169-184.

Gidenne T., Lebas F., 2006. Feeding behaviour in rabbits. In: Feeding in domestic vertebrates. From structure to behaviour. Bels V.L. (Ed), CABI Publishing, Wallingford, UK, 179209.

Gidenne T., Jehl N., Ségura M., MichaletDoreau B., 2002. Microbial activity in the caecum of the young rabbit: impact of a dietary fibre deficiency and of intake level. Anim. Feed Sci. Technol., 99, 107-118.

Gottlieb G. 1971. Ontogenesis of sensory function in birds and mammals. In: The biopsychology of development. Tobach E., Aronson L.R., Shaw E. (Eds), Academic Press, New York, USA, 67-282.

Gyarmati T., Szendro Z., Maertens L., BiroNémeth E., Radnai I., Milisits G. Matics Z., 2000. Effect of suckling twice a day on the performance of suckling and growing rabbits. Proc. $7^{\text {th }}$ World Rabbit Congr., July, Valencia, Spain, C, 283-290.

Hudson R., 1993. Rapid odour learning in newborn rabbits: connecting sensory input to motor output. Ger. J. Psychol., 17, 267-275.

Hudson R., Distel H., 1982. The pattern of behaviour of rabbit pups in the nest. Behaviour, 79, 255-271.

Hudson R., Distel H., 1983. Nipple location by newborn rabbits: evidence for pheromonal guidance. Behaviour, 82, 260-275.

Hudson R., Garay-Villar E., Maldonado M., Coureaud G., 2003. Rabbit pups can orient to the nest by smell from birth. Chem. Senses 28, 551.

Ivanistkii A.M., 1962. The morphophysiological investigation of development of conditioned alimentary reactions in rabbits during ontogenesis. In: Experimental studies of higher nervous activity in man and animals, Works of the Institute of Higher Nervous Activity, Moscow, Physiological series, Israel program for scientific translations Ltd, 4, 126-141.

Jilge B., 1993. The ontogeny of circadian rhythms in the rabbit. J. Biol. Rhythms, 8, 247260.

Keil W., Von Stralendorff F., Hudson R., 1990. A behavioral bioassay for analysis of rabbit nipplesearch pheromone. Physiol. Behav., 47, 525-529.

Kindermann U., Gervais R., Hudson R., 1991. Rapid odor conditioning in newborn rabbits: Amnesic effect of hypothermia. Physiol. Behav., 50, 457-460.

Kovacs M., Szendrõ Z., Csutoras I., Bota B., Bencsne K.Z., Orova Z., Radnai I., Birone N.E. Horn P., 2004. Development of the caecal microflora of newborn rabbits during the first ten days after birth. In Proc. $8^{\text {th }}$ World Rabbit Congr., September, Puebla, Mexico, 5, 1091-1096.

Langenbach G., Brugman P., Weijs W.A., 1992. Preweaning feeding mechanisms in the rabbit. J. Dev. Physiol., 18, 253-261.
Lebas F., 1972. Effet de la simultanéité de la gestation et de la lactation sur les performances laitières chez la lapine. Ann. Zootech., 21, 129131.

Maertens L., Villamide M.J., 1998. Feeding systems for intensive production. In: The nutrition of the rabbit. De Blas C., Wiseman J. (Eds), CABI publishing, Wallingford, UK, 255-271.

Maertens L., Lebas F., Szendro Zs., 2006. Rabbit milk: a review of quantity, quality and non dietary affecting factors. World Rabbit Sci., $14,205-230$

Mirabito L., Galliot P., Souchet C., 2004. Effet de la surface disponible et de l'aménagement des cages sur les performances zootechniques et le comportement des lapines et des jeunes. Proc. Journ. Nat. Elevage du lapin de chair, Novembre, Paris, France, 40-52.

Moncomble A.S., Quennedey B., Coureaud G., Langlois D., Perrier G., Schaal B., 2004. Newborn rabbit attraction toward maternal faecal pellets. Dev. Psychobiol., 45, 277.

Moncomble A.S., Coureaud G., Quennedey B., Langlois D., Perrier G., Brossut R., Schaal B., 2005. The mammary pheromone of the rabbit: where does it come from? Anim. Behav., 69, 2938 .

Montigny D., Coureaud G., Schaal B., 2006. Newborn rabbit response to the mammary pheromone: from automatism to prandial control. Physiol. Behav., 89, 742-749.

Montigny D., Coureaud G., Schaal B., 2007. Préférences sociales chez le lapin en période de sevrage : impact des apprentissages olfactifs néonatals. 41 emes Coll. Ann. Soc. Fr. Etude du Comportement Animal, Villetaneuse, France, 18-20 avril.

Mykytowycz R., 1968. Territorial marking by rabbits. Sci. Am., 218, 116-126.

Mykytowycz R., Rowley I., 1958. Continuous observations of the activity of the wild rabbit, Oryctolagus cuniculus, during 24-hr periods. CSIRO Wildl. Res., 3, 26-31.

Mykytowycz R., Ward M.M., 1971. Some reactions of nestling of the wild rabbit, Oryctolagus cuniculus (L), when exposed to natural rabbit odours. Forma et Functio, 4, $137-$ 148

Pacheco-Cobos L., Rosetti M., Distel H., Hudson R., 2003. To stay or not to stay: the contribution of tactile and thermal cues to coming to rest in newborn rabbits. J. Comp. Physiol. A, 189, 383-389.

Patris B., Perrier G., Schaal B., Coureaud G., 2008. Early development of filial preferences in the rabbit: implications of nursing- and pheromone-induced odour learning? Anim. Behav., 76, 305-314.

Rödel H.G., Bora A., Kaiser J., Kaetzke P., Khaschei M., von Holst D., 2004. Densitydependent reproduction in the European rabbit: a consequence of individual response and agedependent reproductive performance. Oikos, 104, 529-539.

Rödel H.G., Prager G., Stefanski V., von Holst D., Hudson R., 2008a. Separating maternal and litter size effects on early postnatal growth in two species of altricial mammals. Physiol. Behav., doi:10.1016/j.physbeh.2007.11.047.

Rödel H.G., Hudson R., von Holst D., 2008b. Optimal litter size for individual growth of European rabbit pups depends on their thermal environment. Oecologia, doi:10.1007/s00442008-0958-5.

Scapinello C., Gidenne T., Fortun-Lamothe L., 1999. Digestive capacity of the rabbit during the post-weaning period, according to the milk/solid feed intake pattern before weaning. Reprod. Nutr. Dev., 39, 423-432.

Schaal B., Orgeur P., 1992. Olfaction in utero: can the rodent model be generalized? Quat. J. Exp. Psychol., 44B, 245-278.

Schaal B., Coureaud G., Marlier L., Soussignan R., 2001. Fetal olfactory cognition preadapts neonatal behavior in mammals. In: Chemical signals in vertebrates. MarchlewskaKoj A., Lepri J., Müller-Schwarze D. (Eds), Plenum-Klüwer Academic, New-York, USA, 9 197-205.

Schaal B., Coureaud G., Langlois D., Giniès C., Sémon E., Perrier G., 2003. Chemical and behavioural characterisation of the rabbit mammary pheromone. Nature, 424, 68-72.

Schley P., 1979. Olfaction and suckling behavior in young rabbits. Proc. World Lagomorph Conference, August, Guelph, Canada, 291-294.

Schulte I., Hoy S., 1997. Untersuchungen zum säuge- und saugverhalten und zur mutter-kindbeziehung bei Hauskaninchen. Berl. Münch. Tierärztl. Wschr., 110, 134-138.

Semke E., Distel H., Hudson R., 1995. Specific enhancement of olfactory receptor sensitivity associated with foetal learning of food odours in the rabbit. Naturwissenschaften, 82 148-149.

Serra J, Nowak R., 2005. Développement d'une préférence pour l'environnement postnatal chez le lapereau nouveau-né. 11 èmes Journ. Rech. Cunicole. 29-30 novembre, Paris, France, 11, 41-44.

von Holst D., 1998. The concept of stress and its relevance for animal behaviour. Adv. Stud. Behav., 27, 1-131.

von Holst D., Hutzelmeyer H., Kaetzke P., Khaschei M., Rödel H.G., Schrutka H., 2002. Social rank, fecundity and lifetime reproductive success in wild European rabbits Oryctolagus cuniculus. Behav. Ecol. Sociobiol., 51, 245-254.

Xiccato G., Trocino A., Boiti C., Brecchia G., 2005. Reproductive rhythm and litter weaning age as they affect rabbit doe performance and body energy balance. Anim. Sci., 81, 289-296.

Zarrow M.X., Denenberg V. H., Anderson C.O., 1965. Rabbit: Frequency of suckling in the pup. Science, 150, 1835-1836. 


\section{Résumé}

Aveugles et sourds à la naissance, les lapins nouveau-nés doivent, comme tous les mammifères nouveau-nés, interagir rapidement avec la mère afin de trouver rapidement une tétine et téter. Cela se produit généralement une seule fois par jour, au cours d'une très brève interaction, en conditions d'élevage comme en milieu naturel. Après les 10-15 j qui suivent la naissance, la relation mère-jeunes évolue progressivement, et conduit habituellement au sevrage entre 4 et 6 semaines. Dans ce contexte de développement rapide des jeunes, les femelles allaitantes et les lapereaux ont développé des adaptations sensorielles, physiologiques et comportementales leur permettant de communiquer, et permettant aux jeunes d'ingérer efficacement le lait puis les aliments solides. Ici, nous présentons quelques-unes de ces adaptations, en prenant des exemples d'études expérimentales conduites par différents groupes au cours des dernières décennies. Cet article est une synthèse du chapitre écrit par les auteurs pour le Congrès Mondial de Cuniculture (Coureaud et al 2008b).

\section{Abstract}

The developing rabbit: some data related to the behaviour, feeding and sensory capacities between birth and weaning

Blind and deaf at birth, rabbit newborns need, as all mammal newborns, to rapidly interact with the mother to find the nipples and suck. This usually occurs only once per day, during an interaction drastically limited in time, both in domestic and natural conditions. After days 10-15, the mother-young interactions change progressively, leading to weaning between 4 and 6 weeks. In this context of rapid development of the young, lactating rabbit females and pups have developed some sensory, physiological and behavioural adaptations allowing them to communicate, and allowing the young to ingest milk then solid food efficiently. Here, we present some of these adaptations, taking examples from experimental studies run by several groups during the last decades. This paper is a summary of a chapter and communication recently presented during the last World Rabbit Congress (Coureaud et al 2008b).

COUREAUD G., FORTUN-LAMOTHE L., RÖDEL H.-G., MONCLÚS R., SCHAAL B., 2008. Le lapereau en développement : données comportementales, alimentaires et sensorielles sur la période naissance-sevrage. INRA Prod. Anim., 21, 231-238. 
